Mathematical Modelling and AnAlysis

Volume 21 Number 2, March 2016, 174-187

http://dx.doi.org/10.3846/13926292.2016.1145152

(c) Vilnius Gediminas Technical University, 2016
Publisher: Taylor\&Francis and VGTU

http://www.tandfonline.com/TMMA

ISSN: $1392-6292$

eISSN: $1648-3510$

\title{
Exact and Approximate Analytic Solutions of the Jeffery-Hamel Flow Problem by the Duan-Rach Modified Adomian Decomposition Method
}

\section{Lazhar Bougoffa ${ }^{a}$, Samy Mziou ${ }^{a}$ and Randolph C. Rach}

${ }^{a}$ Al Imam Mohammad Ibn Saud Islamic University (IMSIU)

Faculty of Science, Department of Mathematics, P.O. Box 90950, 11623

Riyadh, Saudi Arabia

$b$ The George Adomian Center for Applied Mathematics

316 South Maple Street, 49057-1225 Hartford, MI, USA

E-mail(corresp.): bougoffa@hotmail.com

E-mail: mzious@imamu.edu.sa

E-mail: tapstrike@gmail.com

Received April 28, 2015; revised January 13, 2016; published online March 15, 2016

\begin{abstract}
This paper aims to find the exact solution in an implicit form for the wellknown nonlinear boundary value problem, namely the MHD Jeffery-Hamel problem, which can be described as the flow between two planes that meet at an angle. Also, two accurate approximate analytic solutions (series solution) are obtained by the variation of the power series method (VPS) and the Duan-Rach modified Adomian decomposition method (DRMA).
\end{abstract}

Keywords: Jeffery-Hamel problem, exact solution, power series, Duan-Rach modified Adomian decomposition method.

AMS Subject Classification: 35A24; 37C10; 49K15; 65L10.

\section{Introduction}

The Jeffery-Hamel problem describes the flow of an incompressible conductive viscous fluid between two rigid planes that meet at an angle $2 \alpha$ as shown in the Figure $1[12,17]$.

The flow can be either diverging or converging, where it is assumed to be purely radial and steady, or $v_{\theta}=v_{z}=0$ and $v_{r}=v_{r}(r, \theta)$ with no-slip conditions at $\theta= \pm \alpha$. Using the continuity and the Navier-Stokes equations in cylindrical coordinates. This leads us to look for solutions of the form

$$
u(r, \theta)=\frac{F(\theta)}{r},
$$




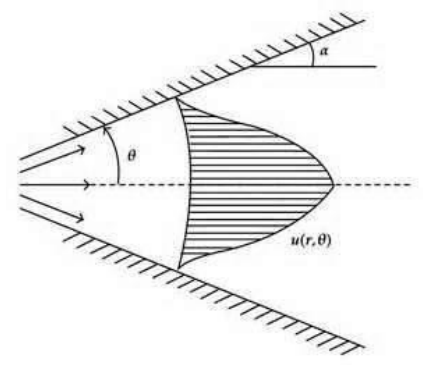

Figure 1. Geometry of the problem

where $F$ is a function of $\theta$ only.

Following [12], we define the dimensionless parameters

$$
f(\eta)=\frac{F(\theta)}{F_{\max }}, \quad \eta=\frac{\theta}{\alpha},
$$

then the problem can be reduced to the solution of the well-known nonlinear third-order differential equation:

$$
f^{\prime \prime \prime}(\eta)+2 \alpha \operatorname{Re} f(\eta) f^{\prime}(\eta)+(4-H) \alpha^{2} f^{\prime}(\eta)=0,
$$

subject to the mixed set of Dirichlet and Neumann boundary conditions

$$
f(0)=1, \quad f^{\prime}(0)=0, \quad f(1)=0,
$$

where $R e$ is the Reynolds number and $H$ is the Hartmann number.

A new exact solution of the Jeffery-Hamel problem in an implicit form was given by Abbasbandy and Shivanian [1], where the authors derived the following equation

$$
\frac{1}{2} f^{\prime 2}(\eta)+\frac{1}{3} \alpha R e f^{3}(\eta)+\frac{1}{2}(4-H) \alpha^{2} f^{2}(\eta)=A f(\eta)+B
$$

which can be written as follows

$$
\left(\frac{d f}{d \eta}\right)^{2}=2 A f+2 B-\frac{2}{3} \alpha R e f^{3}-(4-H) \alpha^{2} f^{2}(\eta) f^{2},
$$

where $A=f^{\prime \prime}(0)+\alpha R e+(4-H) \alpha^{2}$ and $B$ are two constants that satisfy

$$
A+B=\frac{1}{3} \alpha R e+\frac{1}{2}(4-H) \alpha^{2} .
$$

Also, the constants $A$ and $B$, after assuming $\gamma=f^{\prime \prime}(0)$, are given by

$$
A=\gamma+\alpha R e+(4-H) \alpha^{2}, \quad B=-\frac{2}{3} \alpha R e-\frac{1}{2}(4-H) \alpha^{2}-\gamma .
$$

Finally, they obtained a new exact solution in the implicit form

$$
\eta=\int_{f}^{1} \frac{1}{\sqrt{F(\tau)}} d \tau
$$


where

$$
\begin{aligned}
F(\tau)= & 2\left(\left(\gamma+\alpha R e+(4-H) \alpha^{2}\right) \tau-\frac{2 \alpha R e}{3}-\frac{(4-H) \alpha^{2}}{2}-\gamma\right) \\
& -\frac{2 \alpha R e \tau^{3}}{3}-(4-H) \alpha^{2} \tau^{2} .
\end{aligned}
$$

Various analytical approximations methods have also been proposed to solve this problem: the homotopy analysis method (HAM) $[13,14,15,18,19,20,21$, $22]$, the homotopy perturbation method (HPM) [9,10], the optimal homotopy asymptotic method $[9,10,11,16]$ and the Duan-Rach approach (DRA) [5], where the authors proposed a new modified recursion scheme for the resolution of such boundary value problems for nonlinear ordinary differential equations. They derived the following recursion scheme for the solutions components:

$$
\left\{\begin{array}{l}
f_{0}(\eta)=1-\eta^{2} \\
f_{n+1}(\eta)=f_{0}(\eta)+L^{-1} A_{n}(\eta)-\eta^{2}\left[L^{-1} A_{n}(\eta)\right]_{\eta=1}, n \geq 0
\end{array}\right.
$$

where $L^{-1}(\cdot)=\int_{0}^{\eta} \int_{0}^{\eta} \int_{0}^{\eta}(\cdot) d \eta d \eta d \eta$ and $A_{n}(\eta)$ are the Adomian polynomials and obtained from the definitional formula

$$
A_{n}=\frac{1}{n !} \frac{d^{n}}{d \lambda^{n}}\left[N\left(\sum_{i=0}^{n} \lambda^{i} f_{i}(\eta)\right)\right]_{\lambda=0}, n=0,1,2, \cdots
$$

In Dib et al. [5], they used a nocanonical variation of the Duan-Rach modified decomposition method to solve the nonlinear Jeffery-Hamel flow boundary value problem, wherein Dib et al. selected a nocanonical partition of the nonlinear differential equation as $L f(\eta)+N f(\eta)=0$ by incorporating the linear remainder term $R f(\eta)$ into the nonlinear term $N f(\eta)$, which unnecessarily complicates the automated generation of the Adomian polynomials that decompose the nonlinear term $R f(\eta)$. This degrades the efficiency of the subroutines used to calculate the Adomian polynomials tailored to the physical nonlinear term. Also, in Sheikholeslami et al. [23], they combined the classic Adomian decomposition method with the method of undetermined coefficients to solve the nonlinear Jeffery-Hamel flow boundary value problem.

The purpose of our research is to present an exact solution of the Pr. (1.1)(1.2) in an implicit form, and introduce approximate solutions to the JefferyHamel problem by the variation of the power series method $[2,3,4,24]$ and the Duan-Rach modified Adomian decomposition method [6,7,8] and [25]. In our approach (DRAM), we partition the nonlinear differential equation into the canonical operator form of Adomian for the Jeffery-Hamel flow model as $L f(\eta)+R f(\eta)+N f(\eta)=0$, which leads to increased overall efficiency, where $L$ is the linear operator to be inverted, $R$ is the linear remainder operator and $N$ is the nonlinear operator. Finally, we discuss the solutions for different values of the physical parameters of the Jeffery-Hamel equation. 


\section{The exact solution}

Integrating Eq. (1.1) from 0 to $\eta$ and taking into account that $f(0)=1$, we obtain

$$
f^{\prime \prime}(\eta)+\alpha \operatorname{Re} f^{2}(\eta)+(4-H) \alpha^{2} f(\eta)=A,
$$

where $A=\gamma+\alpha R e+(4-H) \alpha^{2}$ and $\gamma=f^{\prime \prime}(0)$. Eq. (2.1) can be written as

$$
f^{\prime \prime}(\eta)+\left(\sqrt{\alpha \operatorname{Re}} f(\eta)+\frac{(4-H) \alpha^{2}}{2 \sqrt{\alpha R e}}\right)^{2}=A+\frac{(4-H)^{2} \alpha^{3}}{4 R e},
$$

or equivalently as

$$
\begin{aligned}
\frac{1}{\sqrt{\alpha R e}} & \left(\sqrt{\alpha R e} f(\eta)+\frac{(4-H) \alpha^{2}}{2 \sqrt{\alpha R e}}\right)^{\prime \prime}+\left(\sqrt{\alpha R e} f(\eta)+\frac{(4-H) \alpha^{2}}{2 \sqrt{\alpha R e}}\right)^{2} \\
& =A+\frac{(4-H)^{2} \alpha^{3}}{4 R e}
\end{aligned}
$$

Let

$$
z(\eta)=\sqrt{\alpha R e} f(\eta)+\frac{(4-H) \alpha^{2}}{2 \sqrt{\alpha R e}} .
$$

Thus Eq. (2.2) becomes

$$
z^{\prime \prime}(\eta)+\sqrt{\alpha \operatorname{Re}} z^{2}(\eta)=B
$$

where $B=\sqrt{\alpha R e}\left[A+\frac{(4-H)^{2} \alpha^{3}}{4 R e}\right]$. Eq. (2.4) is a nonlinear second-order differential equation, and we next derive its implicit solution. Multiplying both sides of Eq. (2.4) by $z^{\prime}(\eta)$, we obtain

$$
z^{\prime}(\eta) z^{\prime \prime}(\eta)=z^{\prime}(\eta)\left(B-\sqrt{\alpha \operatorname{Re}} z^{2}(\eta)\right)
$$

So that

$$
z^{\prime}(\eta) z^{\prime \prime}(\eta) d \eta=\left(B-\sqrt{\alpha R e} z^{2}(\eta)\right) d z
$$

Integrating both sides of Eq. (2.5), we have

$$
\int z^{\prime}(\eta) z^{\prime \prime}(\eta) d \eta=\int\left(B-\sqrt{\alpha \operatorname{Re}} z^{2}\right) d z .
$$

Thus

$$
z^{\prime 2}(\eta)=2\left(B z(\eta)-\frac{\sqrt{\alpha R e}}{3} z^{3}(\eta)\right)+2 c_{1},
$$

where $c_{1}$ is an arbitrary constant of integration. It follows that

$$
z^{\prime}(\eta)= \pm \sqrt{2\left(B z(\eta)-\sqrt{\alpha \operatorname{Re}} z^{3}(\eta) / 3\right)+2 c_{1}} .
$$

Consequently,

$$
\frac{d z}{\sqrt{2\left(B z(\eta)-\sqrt{\alpha R e} z^{3}(\eta) / 3\right)+2 c_{1}}}= \pm d \eta
$$


Therefore,

$$
\int_{0}^{z(\eta)} \frac{1}{\sqrt{2\left(B \tau-\sqrt{\alpha R e} \tau^{3} / 3\right)+2 c_{1}}} d \tau=c_{2} \pm \eta,
$$

where $\tau$ is the variable of integration and $c_{2}$ is another arbitrary constant of integration. In view of (2.3), we have

$$
\begin{aligned}
& z(0)=\sqrt{\alpha R e} f(0)+\frac{(4-H) \alpha^{2}}{2 \sqrt{\alpha R e}}=\sqrt{\alpha R e}+\frac{(4-H) \alpha^{2}}{2 \sqrt{\alpha R e}}, \\
& z^{\prime}(0)=\sqrt{\alpha R e} f^{\prime}(0)=0 .
\end{aligned}
$$

This yields

$$
c_{1}=-B z(0)+\frac{\sqrt{\alpha R e}}{3} z^{3}(0), \quad c_{2}=\int_{0}^{z(0)} \frac{1}{\sqrt{2\left(B \tau-\frac{\sqrt{\alpha R e}}{3} \tau^{3}\right)+2 c_{1}}} d \tau .
$$

Accordingly, the exact solution in the implicit form is therefore given by

$$
f(\eta)=\frac{1}{\sqrt{\alpha R e}} z(\eta)-\frac{(4-H) \alpha}{2 R e}
$$

where $z(\eta)$ is given by

$$
\int_{0}^{z(\eta)} \frac{d \tau}{\sqrt{2\left(\sqrt{\alpha R e}\left[\gamma+\alpha R e+(4-H) \alpha^{2}+\frac{(4-H)^{2} \alpha^{3}}{4 R e}\right] \tau-\frac{\sqrt{\alpha R e}}{3} \tau^{3}\right)+2 c_{1}}}=c_{2} \pm \eta,
$$

and the constant $\gamma$ can be determined by the given boundary condition

$$
z(1)=\sqrt{\alpha R e} f(1)+\frac{(4-H) \alpha^{2}}{2 \sqrt{\alpha R e}}=\frac{(4-H) \alpha^{2}}{2 \sqrt{\alpha R e}} .
$$

This solution is completely different from the one obtained by Abbasbandy and Shivanian [1]. Therefore, it can be regarded as a new expression of the exact solution of the Jeffery-Hamel problem.

\section{The variation of the power series method (VPS)}

The above exact solution in an implicit form is not very convenient for comparison purposes. For that reason, we present here a variation of the the power series method $[2,3,24]$, which can be applied to such equations to obtain accurate quantitative solutions.

Power series solutions of linear homogeneous differential equations in initialvalue problems yield simple recurrence relations for the coefficients but generally are not adequate for nonlinear equations. The variation of the power series method (VPS) using concepts of the decomposition method (the Adomian polynomials $A_{n}$ and transformations of series using the $A_{n}$ ) allows us decreased computation and introduces an effective procedure. 
Integrating Eq. (2.1) from 0 to $\eta$ and taking into account that the initial condition $f^{\prime}(0)=0$, we obtain

$$
f^{\prime}+\alpha \operatorname{Re} \int_{0}^{\eta} f^{2}(\eta) d \eta+(4-H) \alpha^{2} \int_{0}^{\eta} f(\eta) d \eta=A \eta .
$$

Consequently,

$$
f(\eta)=1+\frac{A}{2} \eta^{2}-\alpha R e \int_{0}^{\eta} \int_{0}^{\eta} f^{2}(\eta) d \eta d \eta-(4-H) \alpha^{2} \int_{0}^{\eta} \int_{0}^{\eta} f(\eta) d \eta d \eta .
$$

We look for a series solution defined as

$$
f(\eta)=\sum_{n=0}^{\infty} C_{n} \eta^{n}
$$

Thus

$$
f^{2}(\eta)=\sum_{n=0}^{\infty} A_{n} \eta^{n}=\sum_{n=0}^{\infty} A_{n}\left(C_{0}, C_{1}, \ldots, C_{0}\right) \eta^{n}=\sum_{n=0}^{\infty} \eta^{n} \sum_{k=0}^{n} C_{k} C_{n-k}
$$

which is the same nonlinear term obtained by the power series. The substitution of Eqs. (3.2)-(3.3) into Eq. (3.1) yields

$$
\begin{aligned}
& \sum_{n=0}^{\infty} C_{n} \eta^{n}=1+\frac{A}{2} \eta^{2}-\alpha R e \int_{0}^{\eta} \int_{0}^{\eta} \sum_{n=0}^{\infty} \eta^{n} \sum_{k=0}^{n} C_{k} C_{n-k} d \eta d \eta \\
& \quad-(4-H) \alpha^{2} \int_{0}^{\eta} \int_{0}^{\eta} \sum_{n=0}^{\infty} C_{n} \eta^{n} d \eta d \eta .
\end{aligned}
$$

Carrying out these integrations, we have

$$
\begin{aligned}
& \sum_{n=0}^{\infty} C_{n} \eta^{n}=1+\frac{A}{2} \eta^{2}-\alpha R e \sum_{n=0}^{\infty} \frac{\eta^{n+2}}{(n+1)(n+2)} \sum_{k=0}^{n} C_{k} C_{n-k} \\
& \quad-(4-H) \alpha^{2} \sum_{n=0}^{\infty} C_{n} \frac{\eta^{n+2}}{(n+1)(n+2)} .
\end{aligned}
$$

In the first and second summations on the right, $n$ can be replaced by $n-2$ to write

$$
\begin{aligned}
& \sum_{n=0}^{\infty} C_{n} \eta^{n}=1+\frac{A}{2} \eta^{2}-\alpha R e \sum_{n=2}^{\infty} \frac{\eta^{n}}{n(n-1)} \sum_{k=0}^{n-2} C_{k} C_{n-2-k} \\
& \quad-(4-H) \alpha^{2} \sum_{n=2}^{\infty} C_{n-2} \frac{\eta^{n}}{n(n-1)}
\end{aligned}
$$

Finally, we can equate coefficients of the like powers of $\eta$ on the left side and 
on the right side to arrive at recurrence relations for the coefficients. Thus

$$
\left\{\begin{array}{l}
C_{0}=1, \quad C_{1}=0, \\
C_{2}=\frac{A}{2}-\alpha R e \frac{C_{0}^{2}}{2}-(4-H) \alpha^{2} \frac{C_{0}}{2}=\frac{1}{2}\left(A-\alpha R e-(4-H) \alpha^{2}\right)=\frac{\gamma}{2}, \\
C_{3}=-\frac{\alpha R e}{6} \sum_{k=0}^{1} C_{k} C_{1-k}-(4-H) \alpha^{2} \frac{C_{1}}{6}=0, \\
C_{4}=-\frac{\alpha R e}{12} \sum_{k=0}^{2} C_{k} C_{2-k}-(4-H) \alpha^{2} \frac{C_{2}}{12} \\
\cdots \\
C_{n}=-\frac{\alpha R e}{n(n-1)} \sum_{k=0}^{n-2} C_{k} C_{n-2-k}-(4-H) \alpha^{2} \frac{C_{n-2}}{n(n-1)}, n \geq 4 .
\end{array}\right.
$$

The solution can then be written as

$$
f(\eta)=C_{0}+C_{1} \eta+C_{2} \eta^{2}+C_{3} \eta^{3}+\ldots,
$$

where the only unknown constant is $\gamma=f^{\prime \prime}(0)$. In principle, $\gamma$ can be determined by imposing the boundary condition at the second point, that is $f(1)=0$. Thus, an analytical solution for $f(\eta)$ can be determined by returning the value of $\gamma$ to the truncated solution of the original equation Eq. (3.7).

\section{Approximate analytic solution by the Duan-Rach mo- dified Adomian decomposition method (DRMA)}

We propose here to solve this third-order nonlinear boundary value problem by the Duan-Rach modified Adomian decomposition method (ADM) $[6,7,8]$ and $[25]$.

How does the Duan-Rach modified decomposition method differ from the classic Adomian decomposition method in the context of solving boundary value problems such as the nonlinear Jeffery-Hamel flow boundary value problem? The difference is in the design of the recursion scheme for calculating the solution components which comprise the approximate solutions.

Firstly, in the classic Adomian decomposition method combined with the method of undetermined coefficients (e.g., Appendix A in [6]), the solution components are calculated as if the boundary value problem was an initial value problem with the proviso that the standard Adomian recursion scheme incorporates one or more unknown constants of integration. Thus the classic Adomian recursion scheme results from inserting the decomposition series into an equivalent nonlinear Volterra integral equation. Upon adding these solution components as parameterized by the aforementioned undetermined constants, the remaining boundary conditions are imposed on the approximate solutions resulting in a single or a system of coupled algebraic or transcendental equations of higher and higher degree. The roots of these equations provide more accurate approximations of the undetermined constants and thus more accurate approximate solutions of the original boundary value problem. However, the resulting calculations yield multiple roots for each undetermined constant with the necessity of discarding the spurious roots based upon physical considerations. Secondly, in the Duan-Rach modified decomposition method (e.g., Section 2 in [6]), the unknown constants of integration are determined by formula before the recursion scheme is designed, hence there is no need for 
the determination of multiple roots for each undetermined constant with the concomitant requirement to discard all spurious roots. Thus the Duan-Rach modified recursion scheme results from inserting the decomposition series into an equivalent nonlinear Fredholm-Volterra integral equation, where the nonlinear Fredholm integrals effect the matching of the approximate solutions at the boundaries.

In comparison, in the classic Adomian decomposition method combined with the method of undetermined coefficients to solve nonlinear boundary value problems, we impose the boundary conditions after calculating the solution components $f_{n}(\eta)$, however in the Duan-Rach modified decomposition method to solve nonlinear boundary value problems, we impose the boundary conditions before calculating the solution components $f_{n}(\eta)$.

First, we define the linear operator $L$, linear remainder operator $R$, nonlinear operator $N$ and inverse linear operator $L^{-1}$ as

$$
L f=\frac{d^{3} f}{d \eta^{3}}(\eta), \quad R f=a \frac{d f}{d \eta}(\eta), \quad N f=b f(\eta) \frac{d f}{d \eta}(\eta)
$$

and

$$
L^{-1}(h)=\int_{0}^{\eta} \int_{0}^{\eta} \int_{0}^{\eta} h(\eta) d \eta d \eta d \eta
$$

where $a=(4-H) \alpha^{2}$ and $b=2 \alpha R e$. We rewrite the original equation in Adomian's operator-theoretic notation as

$$
L f(\eta)+R f(\eta)+N f(\eta)=0 .
$$

Then we solve for the term $L f(\eta)$ on the left hand side as

$$
L f(\eta)=-R f(\eta)-N f(\eta) .
$$

Next we integrate both sides of this equation as

$$
L^{-1} L f(\eta)=-L^{-1} R f(\eta)-L^{-1} N f(\eta),
$$

where we calculate

$$
L^{-1} L f(\eta)=\int_{0}^{\eta} \int_{0}^{\eta} \int_{0}^{\eta} \frac{d^{3} f}{d \eta^{3}}(\eta) d \eta d \eta d \eta=f(\eta)-f(0)-\eta f^{\prime}(0)-\frac{\eta^{2}}{2} f^{\prime \prime}(0) .
$$

Upon substitution, we have

$$
f(\eta)-f(0)-\eta f^{\prime}(0)-\frac{\eta^{2}}{2} f^{\prime \prime}(0)=-L^{-1} R f(\eta)-L^{-1} N f(\eta) .
$$

Solving for the solution on the left hand side of this equation, we obtain

$$
f(\eta)=f(0)+\eta f^{\prime}(0)+\frac{\eta^{2}}{2} f^{\prime \prime}(0)-L^{-1} R f(\eta)-L^{-1} N f(\eta) .
$$

Next we substitute the specified boundary values $f(0)=1$ and $f^{\prime}(0)=0$ into Eq. (4.1) and obtain

$$
f(\eta)=1+\frac{\eta^{2}}{2} f^{\prime \prime}(0)-L^{-1} R f(\eta)-L^{-1} N f(\eta) .
$$


Then we evaluate the solution at $\eta=1$ and use the remaining boundary condition to obtain

$$
1+\frac{1}{2} f^{\prime \prime}(0)-L_{1}^{-1} R f(\eta)-L_{1}^{-1} N f(\eta)=0,
$$

where we have defined the definite integral operator $L_{1}^{-1}$ as

$$
L_{1}^{-1} h(\eta)=\left.\left[L^{-1} h(\eta)\right]\right|_{\eta=1}=\int_{0}^{1} \int_{0}^{\eta} \int_{0}^{\eta} h(\eta) d \eta d \eta d \eta
$$

Solving for the second-order derivative evaluated at zero, we deduce

$$
f^{\prime \prime}(0)=-2+2 L_{1}^{-1} R f(\eta)+2 L_{1}^{-1} N f(\eta) .
$$

Upon substitution, we have determined that

$$
f(\eta)=1-\eta^{2}+\eta^{2} L_{1}^{-1} R f(\eta)+\eta^{2} L_{1}^{-1} N f(\eta)-L^{-1} R f(\eta)-L^{-1} N f(\eta),
$$

which is the equivalent nonlinear Fredholm-Volterra integral equation which incorporates all three boundary conditions and thus is without any undetermined constants of integration. By the classic Adomian decomposition method, we decompose the solution into the solution components to be determined by recursion and the nonlinearity into the corresponding Adomian polynomials tailored to the particular nonlinearity as

$$
f(\eta)=\sum_{n=0}^{\infty} f_{n}(\eta), N f=b f(\eta) \frac{d f}{d \eta}(\eta)=\sum_{n=0}^{\infty} A_{n}(\eta),
$$

where the Adomian polynomials are

$$
A_{n}(\eta)=A_{n}\left(f_{0}(\eta), f_{1}(\eta), \ldots, f_{n}(\eta)\right)=\sum_{m=0}^{n} f_{n-m}(\eta) \frac{d f_{m}}{d \eta}(\eta)
$$

Upon substitution, we obtain

$$
\begin{aligned}
\sum_{n=0}^{\infty} f_{n}(\eta)=1 & -\eta^{2}+\eta^{2} L_{1}^{-1} R \sum_{n=0}^{\infty} f_{n}(\eta)+\eta^{2} L_{1}^{-1} \sum_{n=0}^{\infty} A_{n}(\eta) \\
& -L^{-1} R \sum_{n=0}^{\infty} f_{n}(\eta)-L^{-1} \sum_{n=0}^{\infty} A_{n}(\eta) .
\end{aligned}
$$

Then we establish the Duan-Rach modified Adomian recursion scheme as

$$
\left\{\begin{array}{l}
f_{0}(\eta)=1-\eta^{2} \\
f_{n+1}(\eta)=a \eta^{2} \int_{0}^{1} \int_{0}^{\eta} \int_{0}^{\eta} \frac{d f_{n}}{d \eta}(\eta) d \eta d \eta d \eta+b \eta^{2} \sum_{m=0}^{n} \int_{0}^{1} \int_{0}^{\eta} \int_{0}^{\eta} f_{n-m}(\eta) \\
\quad \times \frac{d f_{m}}{d \eta}(\eta) d \eta d \eta d \eta-a \int_{0}^{\eta} \int_{0}^{\eta} \int_{0}^{\eta} \frac{d f_{n}}{d \eta}(\eta) d \eta d \eta d \eta \\
\quad-b \sum_{m=0}^{n} \int_{0}^{\eta} \int_{0}^{\eta} \int_{0}^{\eta} f_{n-m}(\eta) \frac{d f_{m}}{d \eta}(\eta) d \eta d \eta d \eta, \quad n \geq 0 .
\end{array}\right.
$$

Consequently, the first two components of the solution $f(\eta)$ are given as

$$
\begin{aligned}
& f_{0}(\eta)=1-\eta^{2} \\
& f_{1}(\eta)=-\frac{\operatorname{Re} \alpha}{30} \eta^{6}+\left(\frac{\operatorname{Re} \alpha}{6}-\frac{\alpha^{2}(H-4)}{12}\right) \eta^{4}+\left(\frac{\alpha^{2}(H-4)}{12}-\frac{2 R e \alpha}{15}\right) \eta^{2} .
\end{aligned}
$$




\section{Results and comparisons}

In order to verify the accuracy of our present methods, we make a comparison with the well-known results obtained by Abbasbandy and Shivanian [1] and those obtained in [1], numerically by the Runge-Kutta method.

Tables 1 and 2 are the comparison of the approximate analytic solutions obtained by the VPS and DRMA for the $n$ th-stage approximation function of $f(\eta)$, where $n=8$, for different values of Hartmann numbers when $R e=25$ and $\alpha=5^{0}$.

Table 1. Comparison between numerical results [1], the VPS and DRMA solutions for velocity when $\alpha=5^{0}, H=0$ and $R e=25$.

\begin{tabular}{llll}
\hline$\eta$ & Numerical & VPS & DRMA \\
\hline 0 & 1.000000 & 1.0000000000000000 & 1.000000000000000 \\
0.1 & 0.986671 & 0.986455061957507 & 0.986668611341585 \\
0.2 & 0.947258 & 0.946405297103476 & 0.947250391080977 \\
0.3 & 0.883419 & 0.881534702351960 & 0.883403734404411 \\
0.4 & 0.797697 & 0.794426677824558 & 0.797673393063448 \\
0.5 & 0.693233 & 0.688277813748159 & 0.693201028726370 \\
0.6 & 0.573424 & 0.566594550112701 & 0.573387840317988 \\
0.7 & 0.441593 & 0.432970067089886 & 0.441555546599450 \\
0.8 & 0.300674 & 0.291066580785463 & 0.300642848394286 \\
0.9 & 0.152979 & 0.144956035469410 & 0.152959122991207 \\
1 & 0.000000 & 0.000000000000000 & 0.000000000000000 \\
\hline
\end{tabular}

Table 2. Comparison between numerical results [1], the VPS and DRMA solutions for velocity when $\alpha=5^{0}, H=500$, and $R e=25$.

\begin{tabular}{llll}
\hline$\eta$ & Numerical & VPS & DRMA \\
\hline 0 & 1.000000 & 1.000000000000000 & 1.000000000000000 \\
0.1 & 0.990220 & 0.990191088843965 & 0.990221459649174 \\
0.2 & 0.960933 & 0.960817622578632 & 0.960938953460878 \\
0.3 & 0.912273 & 0.912014074665348 & 0.912286635420987 \\
0.4 & 0.844383 & 0.843921028956877 & 0.844404805547638 \\
0.5 & 0.757286 & 0.649683485195127 & 0.757316749874023 \\
0.6 & 0.650719 & 0.522543850373255 & 0.650757737814319 \\
0.7 & 0.523909 & 0.432970067089886 & 0.523952697857051 \\
0.8 & 0.375290 & 0.373693410046810 & 0.375331054503237 \\
0.9 & 0.202125 & 0.200721112552433 & 0.202153222327359 \\
1 & 0.000000 & 0.000000000000001 & 0.000000000000000 \\
\hline
\end{tabular}

The velocity profiles $f(\eta)$ have been plotted in Figures 2-3 for a fixed Reynolds number $(R e=50)$ in two cases, i.e. the convergent channel and divergent channel $\left(\alpha=-5^{0}\right.$ and $\left.\alpha=5^{0}\right)$ flows.

Figure 2 shows the velocity $f(\eta)$ for different increasing values of the Hartmann number $H$ by the VPS method with $\alpha=5^{0}$ and $\alpha=-5^{0}$.

Figure 3 shows the velocity $f(\eta)$ for different increasing values of the Hartmann number $H$ by the DRMA method with $\alpha=5^{0}$ and $\alpha=-5^{0}$. 


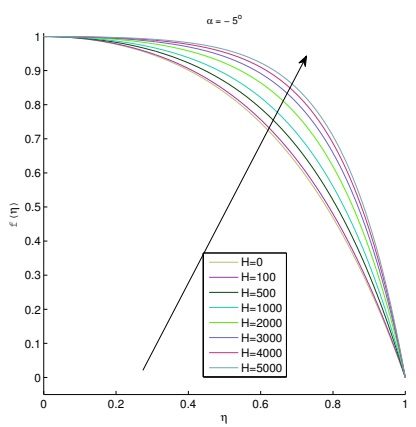

a)

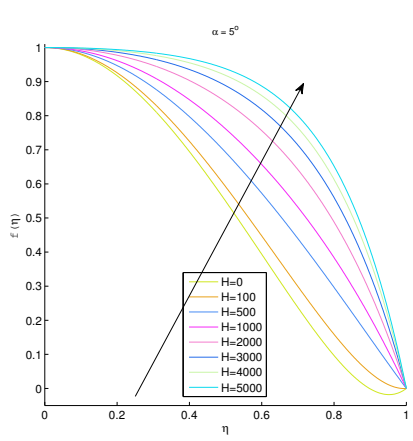

b)

Figure 2. The 8 th-stage approximation obtained by the VPS for $R e=50$ and a) $\alpha=-5^{0}$, b) $\alpha=5^{0}$.

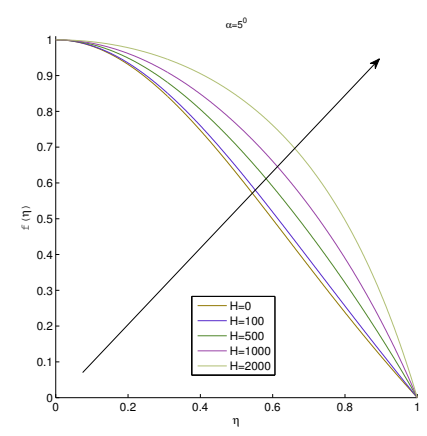

a)

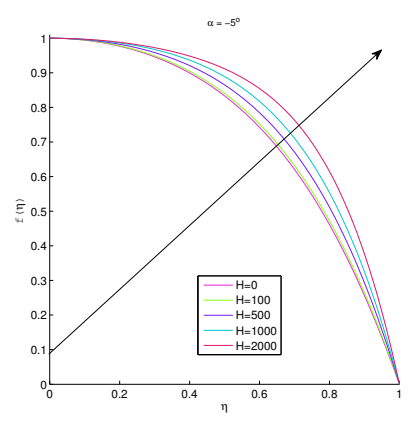

b)

Figure 3. The 8 th-stage approximation obtained by the DRMA for $R e=50$ and a) $\alpha=-5^{0}$, b) $\alpha=5^{0}$.

In all these figures, we observe that when $H$ increases the velocity also increases for both $\alpha=5^{0}$ and $\alpha=-5^{0}$. Moreover when $H$ increases the curvature of the curves increases too.

Table 3 compares the values of $f^{\prime \prime}(0)$ for several values of Hartmann numbers when the Reynolds number is fixed, i.e. $(R e=10)$. Table 5 compares the values of $f^{\prime \prime}(0)$ for different Reynolds numbers $R e$ when $H=0$.

The comparisons in all the above cases are found to be in excellent agreement. In Tables 3 and 4 we compare the values of $\gamma=f^{\prime \prime}(0)$ for different values of Hartmann numbers when the Reynolds number is fixed, i.e. $R e=10$ and $\alpha= \pm 5^{0}$, where the angle $\alpha$ is converted from the unit of degrees to the unit of radians. This clearly indicates that there are good agreements between the numerical and exact solutions. 
Table 3. Comparison between the exact, numerical results [1] and DRMA solutions for $\gamma=f^{\prime \prime}(0)$ for different values of Hartmann numbers when $\alpha=-5^{0}$ and $R e=10$.

\begin{tabular}{llll}
\hline$H$ & Exact & Numerical & DRMA \\
\hline 10 & -1.784546840578866 & -1.78454677 & -1.784546771043338 \\
20 & -1.588153536993253 & -1.58815350 & -1.588153458548008 \\
30 & -1.413692023436293 & -1.41369209 & -1.413691404841239 \\
40 & -1.258993935085547 & -1.25899392 & -1.258987239768864 \\
50 & -1.121989043572515 & -1.12198915 & -1.121950278544477 \\
60 & -1.000742927541422 & -1.00074269 & -1.000580168712826 \\
70 & -0.893474274815973 & -0.89347438 & -0.892933733530960 \\
80 & -0.798567273772039 & -0.79856737 & -0.797045260069311 \\
90 & -0.714567787224302 & -0.71456776 & -0.710794480334909 \\
100 & -0.640177852876257 & -0.64017806 & -0.631716493718889 \\
\hline
\end{tabular}

Table 4. Comparison between the exact, numerical results [1] and DRMA solutions for $\gamma=f^{\prime \prime}(0)$ for different values of Hartmann numbers when $\alpha=5^{0}$ and $R e=10$.

\begin{tabular}{llll}
\hline$H$ & Exact & Numerical & DRMA \\
\hline 10 & -2.251948602981818 & -2.25194858 & -2.251948586581649 \\
20 & -2.527192232687426 & -2.52719225 & -2.527192217906175 \\
30 & -2.832629302137010 & -2.83262931 & -2.832628472744923 \\
40 & -3.169712187544089 & -3.16971221 & -3.169703937108614 \\
50 & -3.539415645558434 & -3.53941564 & -3.539367220445977 \\
60 & -3.942140271189139 & -3.94214029 & -3.941936731143501 \\
70 & -4.377652476579882 & -4.37765249 & -4.376973662025479 \\
80 & -4.845071824815794 & -4.84507184 & -4.843165937552160 \\
90 & -5.342911258048444 & -5.34291127 & -5.338228874413803 \\
100 & -5.869165116815401 & -5.86916513 & -5.858828307218483 \\
\hline
\end{tabular}

\section{Conclusions}

In this work, we have considered the nonlinear Jeffery-Hamel flow problem, which can be described as the flow between two planes that meet at an angle. We have demonstrated that the exact solution can be obtained in a straightforward manner by using a direct method. Also, very good approximate analytic solutions were obtained by the variation of the power series method and the Duan-Rach modified Adomian decomposition method; furthermore a comparison with some well-known results shows that the present solutions are highly accurate.

\section{References}

[1] S. Abbasbandy and E. Shivanian. Exact analytical solution of the MHD Jeffery-Hamel flow problem. Meccanica, 47(6):1379-1389, 2012. http://dx.doi.org/10.1007/s11012-011-9520-3.

[2] G. Adomian. Nonlinear Stochastic Systems Theory and Applications to Physics. Kluwer Academic Publishers, Dordrecht, 1989. http://dx.doi.org/10.1007/97894-009-2569-4. 
[3] G. Adomian. Solving Frontier Problems of Physics: The Decomposition Method. Kluwer Academic Publishers, Dordrecht, 1994. http://dx.doi.org/10.1007/97894-015-8289-6.

[4] L. Bougoffa, R. Rach, A. M. Wazwaz and J. S. Duan. On the Adomian decomposition method for solving the Stefan problem. Internat. J. Numer. Methods for Heat and Fluid Flow, 5(4):912-928, 2015. http://dx.doi.org/10.1108/HFF05-2014-0159.

[5] A. Dib, A. Haiahem and B. Bou-said. An analytical solution of the MHD Jeffery-Hamel flow by the modified Adomian decomposition method. Computers and Fluids, 102(10):111-115, 2014. http://dx.doi.org/10.1016/j.compfluid.2014.06.026.

[6] J. S. Duan and R. Rach. A new modification of the Adomian decomposition method for solving boundary value problems for higher order differential equations. Appl. Math. Comput., 218(8):4090-4118, 2011. http://dx.doi.org/10.1016/j.amc.2011.09.037.

[7] J. S. Duan, R. Rach and A. M. Wazwaz. A reliable algorithm for positive solutions of nonlinear boundary value problems by the multistage Adomian decomposition method. Open Eng., 5(1):59-74, 2014. http://dx.doi.org/10.1515/eng2015-0007.

[8] J. S. Duan, R. Rach, A. M. Wazwaz, T. Chaolu and Z. Wang. A new modified Adomian decomposition method and its multistage form for solving nonlinear boundary value problems with robin boundary conditions. Appl. Math. Modell., 37(20-21):8687-8708, 2013. http://dx.doi.org/10.1016/j.apm.2013.02.002.

[9] M. Esmaeilpour and D.D. Ganji. Solution of the Jeffery-Hamel flow problem by optimal homotopy asymptotic method. Comput. Math. Appl., 59(11):3405-3411, 2010. http://dx.doi.org/10.1016/j.camwa.2010.03.024.

[10] M. Esmaeilpour, D.D. Ganji and E. Mohseni. Application of homotopy perturbation method to micropolar flow in a porous channel. J. Porous Media, 12(5):451-459, 2009. http://dx.doi.org/10.1615/JPorMedia.v12.i5.50.

[11] S.S. Ganji, D.D. Ganji, S. Karimpour and H. Babazadeh. Applications of He's homotopy perturbation method to obtain second-order approximations of the coupled two-degrees-of-freedom systems. Int. J. Nonlinear Sci. Numer. Simul., 10(3):305-314, 2009. http://dx.doi.org/10.1515/IJNSNS.2009.10.3.305.

[12] G. Hamel. Spiralförmige Bewegungen zäher Flüssigkeiten. Jahresbericht der Deutschen Mathematiker-Vereinigung, 25:34-60, 1917.

[13] T. Hayat, M. Khan and M. Ayub. Couette and Poiseuille flow of an Oldroyd 6constant fluid with magnetic field. J. Math. Anal. Appl., 298(1):225-244, 2004. http://dx.doi.org/10.1016/j.jmaa.2004.05.011.

[14] T. Hayat, M. Khan and M. Ayub. On the explicit analytic solutions of an Oldroyd 6-constant fluid. Internat. J. Engrg. Sci., 42(2):123-135, 2004. http://dx.doi.org/10.1016/S0020-7225(03)00281-7.

[15] J.H. He. A coupling method of homotopy technique and perturbation technique for nonlinear problems. Internat. J. Non-Linear Mech., 35(1):37-43, 2000. http://dx.doi.org/10.1016/S0020-7462(98)00085-7.

[16] J.H. He. Homotopy perturbation method for bifurcation of nonlinear problems. Int. J. Nonlinear Sci. Numer. Simul., 6(2):207-208, 2005. http://dx.doi.org/10.1515/IJNSNS.2005.6.2.207. 
[17] G.B. Jeffery. The two-dimensional steady motion of a viscous fluid. Phil. Mag., 29(172):455-465, 1915. http://dx.doi.org/10.1080/14786440408635327.

[18] S.J. Liao. An approximate solution technique not depending on small parameters: a special example. Internat. J. Non-Linear Mech., 30(3):371-380, 1995. http://dx.doi.org/10.1016/0020-7462(94)00054-E.

[19] S.J. Liao. Boundary element method for general nonlinear differential operators. Eng. Anal. Bound. Elem., 20(2):91-99, 1997. http://dx.doi.org/10.1016/S09557997(97)00043-X.

[20] S.J. Liao. On the homotopy analysis method for nonlinear problems. Appl. Math. Comput., 147(2):499-513., 2004. http://dx.doi.org/10.1016/S00963003(02)00790-7.

[21] S.J. Liao and K.F. Cheung. Homotopy analysis of nonlinear progressive waves in deep water. J. Eng. Math., 45(2):105-116., 2003. http://dx.doi.org/10.1023/A:1022189509293.

[22] M. Sajid and T. Hayat. The application of homotopy analysis method to thin film flows of a third order fluid. Chaos Solitons and Fractals, 38(2):506-515, 2008. http://dx.doi.org/10.1016/j.chaos.2006.11.034.

[23] M. Sheikholeslami, D.D. Ganji, H.R. Ashorynejad and H.B. Rokni. Analytical investigation of Jeffery-Hamel flow with high magnetic field and nanoparticle by Adomian decomposition method. Applied Mathematics and Mechanics, 33(1):25-36, 2012. http://dx.doi.org/10.1007/s10483-012-1531-7.

[24] A. M. Wazwaz. Partial Differential Equations and Solitary Waves Theory. Springer Berlin Heidelberg, 2009. http://dx.doi.org/10.1007/978-3-642-002519.

[25] A. M. Wazwaz, R. Rach, L. Bougoffa and J. S. Duan. Solving the Lane-EmdenFowler type equations of higher orders by the Adomian decomposition method. Comput. Model. Eng. Sci., 100(6):507-529, 2014. 